\title{
Enabling Sustainable Improvement in IT Entrepreneurship
}

\author{
Paul E. Renaud, Sheppard D. Narkier, and Sonia D. Bot
}

\author{
"Never confuse movement with action." \\ Ernest Hemingway (1899-1961) \\ Author and journalist
}

\begin{abstract}
Firms must embrace processes that enable the information technology (IT) function to become a strategic partner to the business functions it serves. Process ambidexterity is a way for processes to be augmented to improve alignment and adaptability to new markets and technologies. By applying the principles of process ambidexterity, the key elements required for sustainable change within the capabilities that comprise the IT function of the firm are identified. Furthermore, the scope and depth of the dysfunction that is widespread across large firms that depend upon IT are outlined to provide a contextual basis for presenting a solution framework to address sustainable change. This framework for sustainable change is of primary benefit to IT executives seeking to systematically transform the IT function and enable IT entrepreneurship.
\end{abstract}

\section{Introduction}

Information technology (IT) provides essential services that make it possible for a firm to operate and generate revenue in an increasingly competitive, global marketplace. In many cases, IT is now the electronic face of most firms, as well as the central nervous system. A firm's dependency on IT is a consequence of its need to provide prompt support to its client- and partner-facing business services in response to rapidly shifting global demands. Unfortunately, the IT function often lags behind because it is fearful of the risk of moving too fast and is generally unable to change as quickly as the firm requires. This fear creates a culture that inhibits advanced innovation that can create competitive advantages for the firm.

As firms become increasingly dependent on the capabilities of their IT function, their appetite for change becomes dependent on their ability to accelerate maturity in the IT function. Yet, most firms experience the following pain points:

- Non-IT business executives aggressively introduce "change projects" in reaction to a perceived competitive threat or opportunity. These executives rarely ap- preciate the operational consequences of the new, abrupt changes or the cumulative burden that new capabilities put on the IT function's agility.

- Existing IT capabilities are not explicitly defined and exist at varying levels of maturity. In firms that have a decentralized IT function, capabilities are diffused across isolated pockets, and the same capability can be at different maturity levels in different business units.

- Expertise is not often shared across the firm, because the person or team that possesses the expertise is often too busy to be used by other teams.

- New technology is often introduced and implemented by a team with a very narrow focus that may be indifferent to the broader interests of the firm.

- New processes are often informally derived from ad hoc processes that are not well integrated with other processes.

- Novel technologies that could have a positive impact are rarely proliferated due to inadequate knowledge and support (i.e., supporting competencies), so fear of operational risk often inhibits broader adoption. 


\section{Enabling Sustainable Improvement in IT Entrepreneurship}

\section{Paul E. Renaud, Sheppard D. Narkier, and Sonia D. Bot}

- Costly redundancy occurs when the same new technologies are introduced in disparate and disconnected units of a firm.

- Best practices are rarely implemented even though they are crucial for reducing operational and lifecycle costs.

- A culture of learned helplessness becomes the accepted norm, in which being stuck in the capability trap is widely viewed as unchangeable and inescapable (Repenning and Sterman, 2001; tinyurl.com/m4u77dd).

IT entrepreneurship is the implementation of innovative business-driven practices that better align the IT function to the business functions of the firm. Bot and Renaud (2012; timreview.ca/article/596) established the value of process ambidexterity in improving IT entre- preneurship and established that IT entrepreneurship includes the management of capabilities within IT, as illustrated in Figure 1. This article explores the capability aspect of process ambidexterity to show how a capability-based approach can promote a successful adaptation of innovation that addresses the pain points listed above.

This article defines IT capabilities and presents a framework that senior IT executives can use to improve the IT function's responsiveness to changes in business demand. This framework delineates the capability domains that will need to evolve, while providing a means to introduce changes, ensure implementation, and measure success. This framework leverages existing standards while synthesizing a cohesive approach to continual improvement.

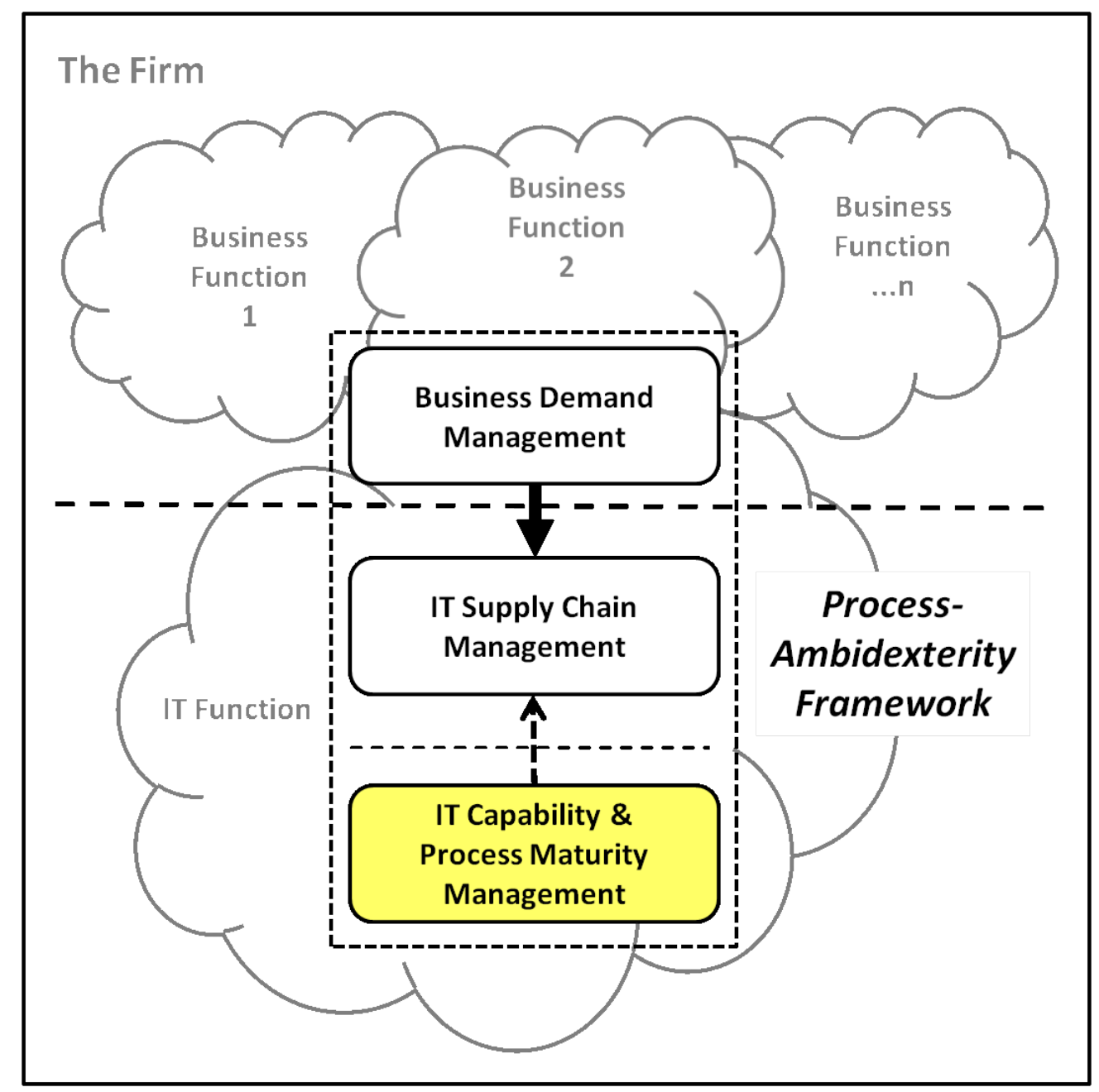

Figure 1. A high-level view of the process-ambidexterity framework 


\section{Enabling Sustainable Improvement in IT Entrepreneurship}

Paul E. Renaud, Sheppard D. Narkier, and Sonia D. Bot

\section{IT Capabilities}

The Open Group (2011; tinyurl.com/3q85nl7) defines a capability as: "an ability that an organization, person, or system possesses." The Capability Model, developed by The Open Group Architecture Framework (TOGAF; togaf.org), decomposes capabilities into:

- technology capabilities that are possessed by a system or tool

- process capabilities that are possessed by an organization

- competency (people skills) capabilities, which are the skills/competencies possessed by a person/team

Most IT organizations have separated IT operations from IT engineering, largely due to the need to improve the cost effectiveness of competency management, and in many cases, these organizations have outsourced some or all of the operational processes and competencies for the same reason. Renaud and Bot (2012; timreview .ca/article/627) examined the impact of outsourcing on process adaptability in the IT supply chain and found that outsourcing generally reduced both business flexibility and IT control, thereby impairing process adaptability. Outsourcing essentially trades off process adaptability in an attempt to gain cost effectiveness.

This article proposes a framework for capability management that promotes process adaptability via the disciplined improvement of capabilities that comprise the maturity of the IT function. Disciplined capability improvement strikes at the pain points identified earlier to deliver cost effectiveness without trading-off process adaptability. In this article, whenever the term "capability" is used by itself, it is understood to refer to all three types of capability: technology, process, and competency).

In many large firms, "learned helplessness" has created an environment in which improvements to any process capability are often viewed as onerous and prohibitive, and therefore are subject to exceptions. Learned helplessness often is the cause of the allergic reaction that many IT functions have to the very practices which would make them more cost effective:

- New functionality is often implemented using noncompliant workarounds that short-cut critical steps that would otherwise enhance reliability.
- Skills augmentation is viewed as an overhead cost when new technology is introduced and consequently is rarely considered strategic, causing new technology capabilities to under under-perform.

- Architecture reviews and standards are often seen as an inhibitor that must be overcome to avoid missing deadlines, and new technology introduction is often forced, which can sometimes result in severe, unintended consequences.

As a result, much of the firm's investment in new technology does not meet expectations because it takes too long for the investment to be scaled out and made more robust. New technologies and the expertise to exploit them often remain in isolated pockets within the firm. If the technology is attractive enough, multiple business functions will deploy and maintain their own versions, proliferating inefficiencies and wasting work.

Technology capabilities can certainly solve complexity, reduce costs, and promote agility, but the IT function must also advance skill maturity and enable those skills to operate efficiently by promoting process maturity. Implementing technology alone will do little and will be rapidly abandoned if supporting skills are not developed and if the new capability is not integrated into existing processes. Furthermore, new technologies often require new or modified processes for them to be accepted within the constraints of existing organizations.

Failure to fully implement process and skill changes necessitated by introducing new technology will inevitably result in the new technology being too expensive to employ. Sustainable change requires that all three capability types are improved in a balance with each other, often as a composite capability that combines improvements in process and competency capabilities along with advances in technology capabilities.

\section{Sustaining Entrepreneurial Change in IT}

Most people view maturity as the end goal of a one-way journey. Yet, the most important and counterintuitive concept that IT professionals struggle with is the fact that maturity in IT capability is not static; it can degrade as well as improve. IT maturity is fluid because the business it supports must be agile in the face of market forces and regulatory forces and, consequently, is always changing. In addition, the introduction of new technology capabilities occurs at a pace that outruns the ability of most IT organizations to absorb these cap- 


\section{Enabling Sustainable Improvement in IT Entrepreneurship}

\section{Paul E. Renaud, Sheppard D. Narkier, and Sonia D. Bot}

abilities through adjustments in processes and competencies needed to embrace these innovations properly. Carnegie Mellon's Software Engineering Institute (SEI; sei.cmu.edu) has defined five progressive stages of process maturity (tinyurl.com/7pa6b), which are useful for assessing and improvings existing IT process maturity but do not consider changes to the scope of maturity required (e.g., new processes) or the possibility of maturity erosion in existing processes. Firms with large IT organizations will see degradation in their maturity levels over time unless specific effort is made to halt the erosion. A key consideration is the level of investment and degree of risk the IT function is willing to incur to reach the maturity level they need to enable their strategy.

Renaud and Bot (2012; timreview.ca/article/626) identified that IT entrepreneurship can occur even if the firm itself is non-entrepreneurial, but IT entrepreneurship cannot be a one-time event because the IT function must remain continuously aligned to the continually changing needs of the firm. IT entrepreneurship requires a sustainable governance ability that ensures dynamic and continuous alignment.
Creating an IT function that is continuously aligned to the needs of the firm requires a significant shift in mindset of most IT executives. Specifically, IT entrepreneurship requires a deliberate, collaborative effort that leverages existing capabilities to implement change such that the changes are widely and rapidly adopted. Advancing technology, process, and competency capabilities in concert with each other is essential for attaining rapid and wide-scale adoption.

Coordinating capability advancement across the wide breadth of capabilities in an IT function is a large undertaking, which can be greatly simplified by exploiting the fact that capabilities can be combined with other capabilities to create higher-level capabilities. Figure 2 illustrates, for example, that the process capability for system monitoring can be combined with the technology capabilities of security and internet-protocol connection monitoring and the process capability for patch-level monitoring to create a higher-level capability called server health monitoring.

In fact, it is the combination of process and technology capabilities that makes such advancement feasible, for

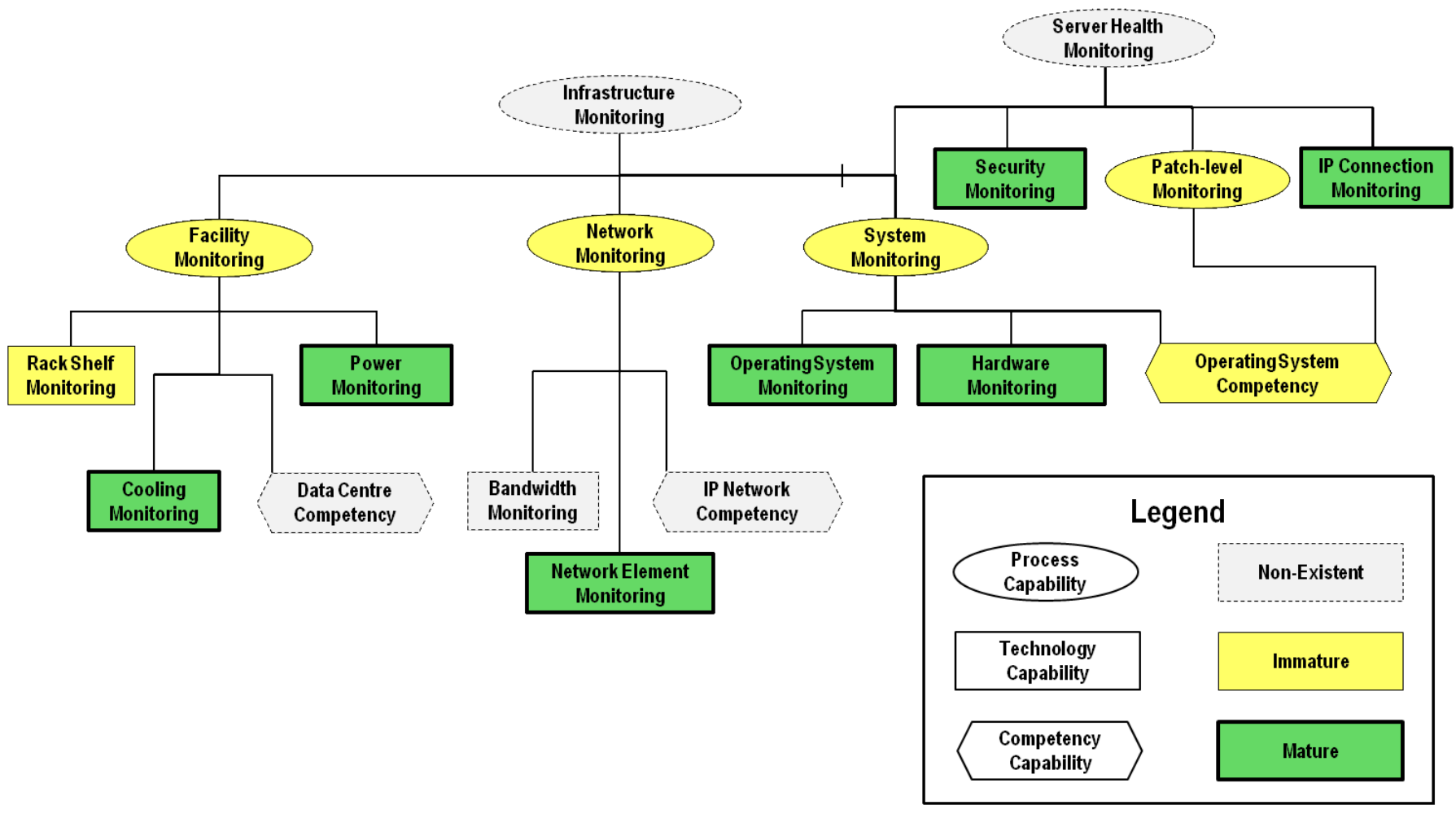

Figure 2. An example capability hierarchy 


\title{
Enabling Sustainable Improvement in IT Entrepreneurship
}

\author{
Paul E. Renaud, Sheppard D. Narkier, and Sonia D. Bot
}

neither type of capability would be sufficient if implemented alone. Observe that a process capability always contains at least one competency capability or is constituted from at least one subordinate process capability. A process would have to be fully automated not to employ at least one competency or skill, and alternatively, if it were fully automated, the automation technology would be a technology capability.

In essence, capabilities are the building blocks for sustainable change within the IT function. Any change in the IT function requires the development or implementation of one or more capabilities. Disciplined, systematic, and sustained improvement in capabilities is fundamental to improving an IT function's ability to change to meet the firm's stated strategic goals. Explicitly mapping of capabilities, their dependencies, and relative maturity levels is the key to sustaining entrepreneurial change.

\section{Coordinated Improvement of Capability Maturity}

The overall maturity of the IT function can be measured by the combination of the number of capabilities implemented and the relative maturity of each capability. The more capabilities that an IT function possesses and the more mature each of those capabilities are, the more mature and adaptable the overall IT function will be. Greater maturity in the IT function promotes greater agility, alignment, and responsiveness to the business.

In practice, the amount of change that is possible within the IT function in any given year is constrained by other priorities that may make improvements too risky to attempt, as well as by the degree of difficulty in changing culture and behaviour within the IT function. Given that IT maturity improvement depends both on the IT function's goals (new capabilities) and capacity for change (introducing and improving capabilities), both must be grounded in the context of business needs if the ability to change is to keep up with the goals that are driving change. Hence, sustainable change is an enabler for IT maturity and the lack of sustainable change limits a firm's capacity for improving IT maturity.

Identifying, managing, and defining capabilities are the first phases of capability maturity improvement. A disciplined basis for capability improvement provides clear objectives, measurable criteria and lifecycle perspective. Bot and Renaud (2012; timreview.ca/article/596) identified that process ambidexterity requires a processmanagement control system to provide this discipline.
The scope and function of this system needs to be generalized to control improvement across the entire range of capabilities, including people and technology, in addition to controlling the improvement of process capabilities. In other words, sustainable improvement in competency capabilities and technology capabilities need to be managed as processes if the IT function is to mature. Furthermore, the systematic addition and improvement of capabilities also needs to be managed as a process to sustain improvements in the firm's capabilities. Thus, the same mechanisms commonly used for process maturity improvement can be applied more generally to the challenge of capability maturity improvement, which encompasses technology, process, and competency capabilities.

By viewing IT maturity improvement as a set of processes with clear stages and tasks to accomplish, a management system can be defined as illustrated in Figure 3 . This management system requires two levels to ensure that the advancement of capabilities is coordinated, across the three types of capabilities as well as within each type of capability.

In Figure 3, the top-level process focuses on how to coordinate the addition of new capabilities in the context of existing ones and how to prioritize which immature capabilities to improve based on the dependencies of the new capabilities being added. This process provides clear guidance on how to measure capabilities and delivers a periodic plan that decides which capabilities are to added or replaced and which need improvement based upon business goals and the appetite for investment in change. By identifying foundational and requisite missing capabilities and setting objectives for capability improvement that closes those gaps, IT maturity is accelerated in a manner that is cost-effectively aligned to the firm's needs.

The IT Capability Maturity Improvement process is the control process by which planned improvements in different capabilities can be prioritized and tracked. This process should be invoked on an event-driven basis whenever new capabilities are to be added, and on a periodic-basis to review the status of capability maturity improvement initiatives and their key performance indicators (KPIs). One of the outputs of this process is a balanced scorecard for how the IT function is maturing.

Implementing capability maturity improvement for specific technology, process, or competency capabilities requires initiation and coordination of specialized improvement processes that operate on the lower level 


\section{Enabling Sustainable Improvement in IT Entrepreneurship}

\section{Paul E. Renaud, Sheppard D. Narkier, and Sonia D. Bot}

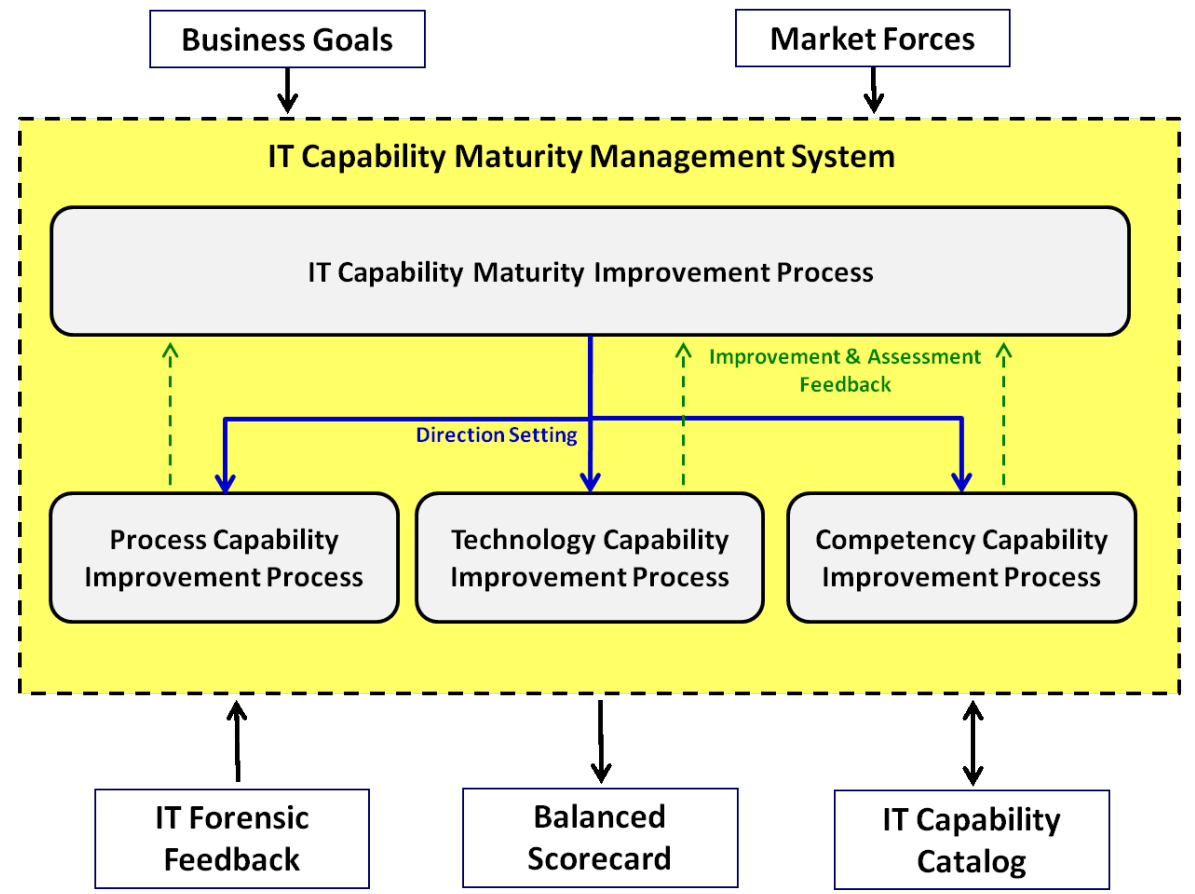

Figure 3. A management system for sustainable IT capability maturity improvement

of the maturity-management system. These improvement processes are specialized for the type of capability being improved. For example:

- Incremental improvement of specific technology capabilities is best performed by a Technology Capability Improvement process that can be as defined in Table 1c below or, in a more mature IT function, based on an IT Solutions Lifecycle Management process that coordinates with IT Product and Standards Management processes described by Renaud and Bot (2012; timreview.ca/article/626).

- Similarly, Process Capability Improvement is best performed by a disciplined and systematic methodology for managing the business to improve customer satisfaction and bottom line results, such as Lean Six Sigma (tinyurl.com/cy9nbfw). Lean Six Sigma is a popular and effective methodology for process improvement that applies a discipline of defining, measuring, analyzing, improving, and controlling processes as shown in Table $1 \mathrm{~b}$ below. Other methodologies for process improvement could also be employed.

- Competency capability improvement can be managed by the disciplined application of the SEI People Capability Maturity Model (tinyurl.com/l8lfch2), as defined in Table 4 below.
The operating framework for each the three types of capability improvement processes has five similar stages with unique tasks for each stage that are appropriate for that capability type. The overall Capability Maturity Improvement Process utilizes these same five stages and also has unique tasks. All four processes, their stages, and unique tasks are delineated in Table 1.

\section{Measuring Sustainable Change}

As the maturity of the IT function improves, the quality of information on the performance of its processes and its ability to improve in response to business needs also improves. The lower-level improvement processes should produce both predictive as well as outcome-oriented KPIs that measure capability maturity improvement so that the top-level process can prioritize which capabilities need subsequent improvement.

Improving IT cost-effectiveness requires insight into how the IT function is improving. Senior management should review scorecards for maturity improvement that are produced by the system for capability maturity management and judge how best to guide investments to achieve their goals. Improved accountability is a natural by-product of a disciplined approach to capability improvement because improvements can be planned and measured on a recurring basis. This improved 


\section{Enabling Sustainable Improvement in IT Entrepreneurship}

\section{Paul E. Renaud, Sheppard D. Narkier, and Sonia D. Bot}

Table 1. The Capability Maturity Improvement Process

\section{A. IT Capability Maturity Improvement Process}

\begin{tabular}{|l|l|l|l|l} 
Define & Measure & Analyze & Improve & Control \\
\hline $\begin{array}{l}\text { Review demand for new } \\
\text { capabilities }\end{array}$ & $\begin{array}{l}\text { Review performance of } \\
\text { existing capabilities and } \\
\text { update maturity level } \\
\text { within the capability } \\
\text { Map capability } \\
\text { dependencies for new } \\
\text { capabilities }\end{array}$ & $\begin{array}{l}\text { Weight weak capabilities } \\
\text { by number of capabilities } \\
\text { depending on them }\end{array}$ & $\begin{array}{l}\text { Review demand for new } \\
\text { capabilities }\end{array}$ & $\begin{array}{l}\text { Review performance } \\
\text { of existing } \\
\text { capabilities and } \\
\text { update maturity level } \\
\text { within the capability } \\
\text { hierarchy }\end{array}$ \\
$\begin{array}{l}\text { Create balanced } \\
\text { scorecard for } \\
\text { communicating overall } \\
\text { capability maturity }\end{array}$ & & $\begin{array}{l}\text { Papabilities to improve } \\
\text { capect }\end{array}$ & & \\
\hline
\end{tabular}

\section{B. Process Capability Improvement Process}

\begin{tabular}{|c|c|c|c|c|}
\hline Define & Measure & Analyze & Improve & Control \\
\hline $\begin{array}{l}\text { Define problem } \\
\text { statement, process } \\
\text { objectives, target } \\
\text { customers, and } \\
\text { ownership } \\
\text { Identify outcome KPI }\end{array}$ & $\begin{array}{l}\text { Capture current state for } \\
\text { existing process } \\
\text { Capture customer } \\
\text { requirements for process } \\
\text { and prioritize } \\
\text { Measure process } \\
\text { capability and stability } \\
\text { Establish improvement / } \\
\text { performance targets }\end{array}$ & $\begin{array}{l}\text { Identify and validate with } \\
\text { data root causes for } \\
\text { failures, variability, and } \\
\text { waste (existing processes) } \\
\text { Select high-level design } \\
\text { based on analysis of } \\
\text { alternatives (new } \\
\text { processes) } \\
\text { Quantify opportunity } \\
\text { area }\end{array}$ & $\begin{array}{l}\text { Evaluate and prioritize } \\
\text { alternative solutions } \\
\text { Design, pilot, and } \\
\text { validate process } \\
\text { improvements / new } \\
\text { process } \\
\text { Implement } \\
\text { improvements / new } \\
\text { process }\end{array}$ & $\begin{array}{l}\text { Institutionalize } \\
\text { improvements / new } \\
\text { process (wide scale) } \\
\text { Implement Process } \\
\text { Management Control } \\
\text { System for ongoing } \\
\text { monitoring and } \\
\text { control }\end{array}$ \\
\hline
\end{tabular}

\section{Technology Capability Improvement Process}

\begin{tabular}{|l|l|l|l|l|} 
Define & Measure & Analyze & Improve & Control \\
\hline Identify scope and needs & Assess alternatives & Pilot preferred solution & $\begin{array}{l}\text { Implement required } \\
\text { training }\end{array}$ & $\begin{array}{l}\text { Implement } \\
\text { performance } \\
\text { mefine criteria for } \\
\text { evaluating alternatives }\end{array}$ \\
$\begin{array}{l}\text { Examine make / buy / } \\
\text { sourcing factors }\end{array}$ & Assess trade-offs (ATAM) & Assess missing kills & $\begin{array}{l}\text { Implement supporting } \\
\text { processes } \\
\text { Assess changes to } \\
\text { processes }\end{array}$ & $\begin{array}{l}\text { Assess technology } \\
\text { maturity level }\end{array}$ \\
\end{tabular}

\section{Competency Capability Improvement Process}

\begin{tabular}{|l|l|l|l|l|} 
Define & Measure & Analyze & Improve & Control \\
\hline $\begin{array}{l}\text { Define scope and } \\
\text { supporting skills }\end{array}$ & $\begin{array}{l}\text { Identify linkage to } \\
\text { processes }\end{array}$ & Assess existing skills base & $\begin{array}{l}\text { Implement required } \\
\text { training }\end{array}$ & $\begin{array}{l}\text { Implement skills } \\
\text { tracking }\end{array}$ \\
$\begin{array}{l}\text { Define criteria for } \\
\text { attainment }\end{array}$ & $\begin{array}{l}\text { Implement skills testing } \\
\text { or survey } \\
\text { Define KPIs }\end{array}$ & $\begin{array}{l}\text { relevant process areas } \\
\text { Implement } \\
\text { compliance in role } \\
\text { monitoring }\end{array}$ & & Assess competency \\
maturity level
\end{tabular}




\section{Enabling Sustainable Improvement in IT Entrepreneurship}

Paul E. Renaud, Sheppard D. Narkier, and Sonia D. Bot

accountability causes the IT function to become more a proactive in improvement instead of being a reluctant participant that is driven by change.

Although all aspects of the IT Supply Chain must be measured including IT maturity, in the authors' experience, most IT functions collect and track too many metrics yet somehow fail to collect the most useful ones. This inefficiency adds unnecessary cost and diminishes the value and usefulness of producing KPIs for management purposes. This section provides criteria for selecting meaningful KPIs for a balanced understanding of capability maturity.

The method for determining the maturity of a capability varies by the capability type. For example a technology capability is measured differently than a process or competency capability. In general, capabilities can be measured by their suitability (i.e., fit for purpose), the extent to which they are (appropriately) employed (i.e., scope), as well as the "depth of effectiveness" of the capability itself. For example:

- The maturity of a technology capability suitable for a specific need can be measured by the combination of the depth of its features as well as how widely this technology is in use within the firm.

- A process capability can be measured using a combination of two criteria: i) the maturity and stability of the process in terms of how effective it is in meeting the needs of the firm and ii) how consistently the process is used across the firm.

- A competency capability can be measured by its appropriateness within a process step, the depth of mastery of the skills involved, and the number of individuals in the same role that possess those skills.

Understanding the maturity of the IT function requires that capability assessments are performed on a periodic basis so that opportunities to improve are identified. These assessments should be based on objective criteria that measure each capability's proliferation, appropriate usage, and effectiveness in fulfilling its intended purpose. An important outcome is the formal definition of existing capabilities and how they related to each other. This definition forms the basis for an annual capability-improvement plan to advance maturity levels on a sustainable basis.
Bot and Renaud (2012; timreview.ca/article/596) identified that successful capability management requires establishing sub-level KPIs. Although KPIs must be measurable, it is important to recognize that they can be categorical as well as quantitative metrics. Bot and Renaud highlighted that seemingly more precise quantitative metrics (i.e., continuous interval or ratio quantities) can often be less accurate than categorical (i.e., discrete qualitative) metrics. Furthermore, metrics should be both predictive as well as based on outcomes.

Implementing accurate process-ambidexterity metrics requires a balanced definition of both predictive and outcome metrics in the following performance domains:

1. Fit for purpose: meets defined requirements, improves existing IT solutions, and assures responsiveness to the needs of the firm. Metrics in this measurement domain predicatively measure process alignment, focusing primarily on the responsiveness and agility aspects of process adaptability. When applied at a process or project level, sub-level KPIs assess whether business priorities and service levels are met (i.e., they determine whether it meets, or is on-track to meet its objectives - ensuring an emphasis on outcomes instead of self-absorbed activities performed). Overby (2011; tinyurl.com/3hw8z7y) identified that meeting objectives is only a pre-condition for sustainable IT because the IT function must also be responsive to the business. Hence, a key aspect of KPIs designed to measure the fit for purpose is measuring the rate at which a project or process meets its objectives.

2. Feasibility: identifies and scopes the changes needed in IT to support the new technology. To best assure process customization, metrics in this measurement domain should address all three capability areas. These metrics focus on the customization aspect of process adaptability as well as indirectly gauging the internalalignment aspect of process alignment. When applied at the level of a process or project, these sub-level KPIs assess the feasibility of how project scope, completion, and risk management are evaluated. For in-flight projects, these KPIs measure progress towards completion of the feasible scope (i.e., they determine whether it is feasible for a given project to be completed, thereby preventing situations where a project never ends). For processes, these KPIs measure the predictive and outcome controls for the process (i.e., they determine whether the process is operating within its defined 


\section{Enabling Sustainable Improvement in IT Entrepreneurship}

\section{Paul E. Renaud, Sheppard D. Narkier, and Sonia D. Bot}

parameters, thereby preventing out-of-control processes or processes from which there is no escape).

3. Effectiveness: identifies value-added aspects or removal of non-value-adding activities. For instance, is the business case realistic given the investment that the firm is prepared to make? Does the change introduce risks or trade-offs that are acceptable to the firm? Are non-value-adding activities and waste minimized? Metrics in this measurement domain enable practitioners to balance the practices that are being implemented to ensure that they are adequate as well as lean. These metrics focus on the flexibility and responsiveness aspects of process adaptability as derived outcome metrics of process alignment. These sub-level KPIs measure expected or added value given the investment (i.e., they determine whether a process or project adds value, thereby forcing accountability and a willingness to change to more effective alternatives).

Measurement has a well-accepted role within the IT function, but traditional approaches to measurement rarely consider whether the IT function is meeting its business goals; in essence, they do not measure why change is occurring. Instead, most IT functions calculate hundreds of metrics pertaining to the implementation of change (i.e., what, when, and how) that are rarely employed by senior business management because there is no apparent relevance to the "why questions". Given that metric collection, analysis, and interpretation is expensive, the IT function should seek to minimize KPIs without sacrificing balance and accuracy and without losing the benefits provided by implementation-oriented measurement of change.

This balance can be accomplished by applying the above KPI-measurement areas evenly for each new project and process using the paradigm of a measurement spirit level (tinyurl.com/5e4d4n), as illustrated in Figure 4. In a spirit level, a bubble floats to indicate the direction that the level is unbalanced. When the bubble is centered in the level, the item being measured is perfectly balanced. The three-way measurement of feasibility, effectiveness, and fit for purpose promotes a culture of relevance within the IT function. Too many metrics in one vertex means that measurement of the other vertices is weaker and is unlikely to effectively support business goals.
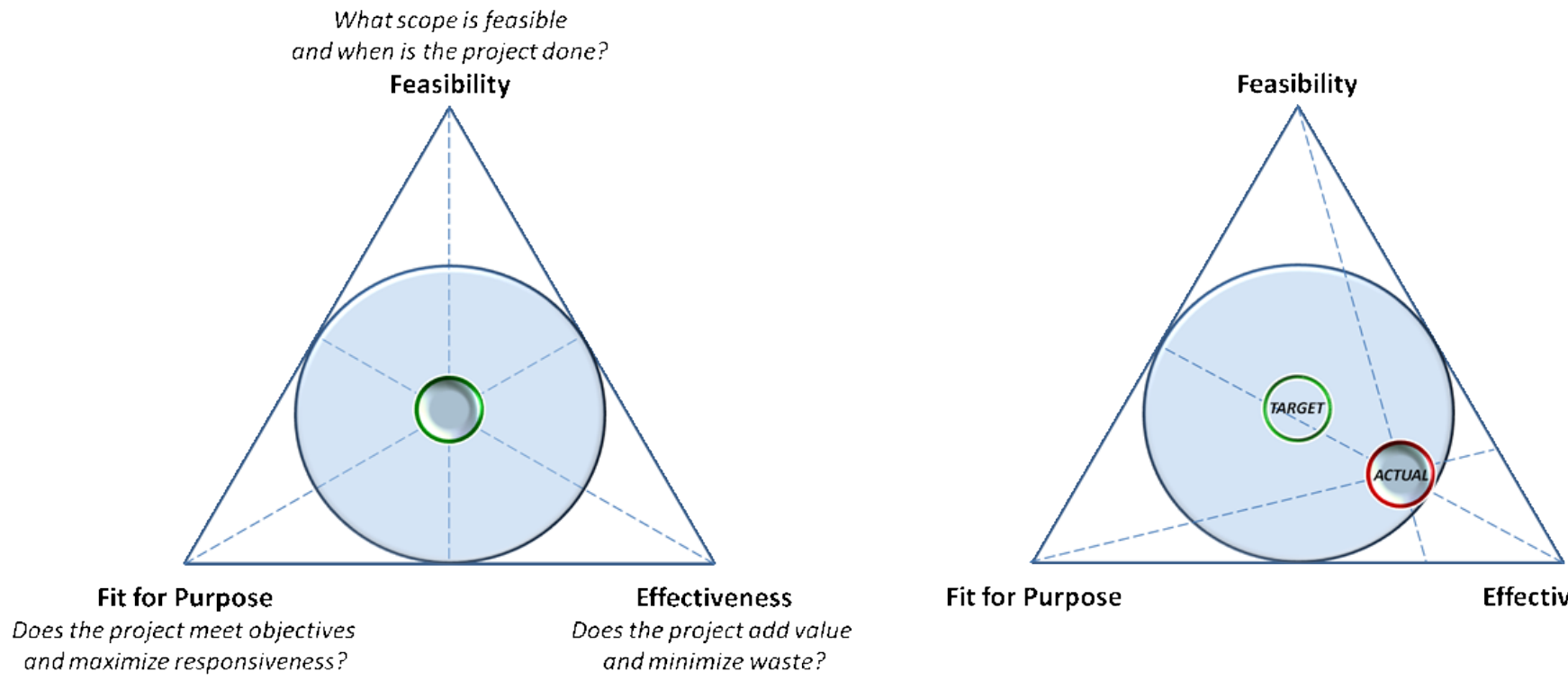

Fit for Purpose

Effectiveness

Figure 4. A spirit level for sustainable metrics (Left: an ideal/balanced scenario; Right: an unbalanced scenario where improvement is required) 


\title{
Enabling Sustainable Improvement in IT Entrepreneurship
}

\author{
Paul E. Renaud, Sheppard D. Narkier, and Sonia D. Bot
}

\section{Conclusion}

IT entrepreneurship can be a cornerstone of a firm's competitive success in a global, highly connected marketplace where agility, adaptation, and alignment are necessary. In summary, accomplishing and sustaining IT entrepreneurship requires:

1. Systematic, maturity-driven, and cost-effective investment in IT maturity improvement. This investment must be supported by executive sponsorship in technology, new processes, skills, and competencies to continuously introduce innovation, which is vital to a firm's competitive success.

2. Creation of the cultural environment for IT to become a strategic enabler of change. Unlike other forms of entrepreneurship, IT entrepreneurship must be continuously aligned and change must be proactively managed. These changes must occur in the context of IT maturity improvement for IT entrepreneurship to be sustainable.

3. Understanding IT maturity through the lens of three types of capability maturity improvement: technology, process, and competency. Composite capabilities consisting of these three types will be assembled to meet specific business needs and to advance IT maturity. The resulting hierarchy of capabilities is self-describing and provides a useful taxonomy for communicating scope and interdependencies, as well as maturity.

4. Recognition that IT maturity is as dynamic as the capabilities required to enhance the firm's performance in a changing global economy. The scope of IT maturity is continually growing and overall maturity can erode and, in fact, degrade whenever new capabilities depend on immature existing capabilities.

5. Acceptance that the introduction of a new technology capability by itself is not sustainable. And, although enterprise architecture and blueprints are essential for the successful adoption of new technology, it is insufficient on its own for sustaining technology change. New technologies also need to be balanced and supported by appropriate competen- cies and processes. A capability hierarchy is a useful tool for understanding IT maturity and mapping the interdependency between capabilities of all types.

6. Managing IT maturity improvement by using an integrated capability maturity management system. Sustainable maturity improvement requires a twolevel management system encompassing capability maturity and individual capability improvement by type of capability.

7. Appreciating that a unified maturity improvement system, based on Lean Six Sigma, can improve all three types of capability: technology, competency, and process. The use of a common system facilitates management and promotes sustainable governance of change.

8. Minimizing KPIs without sacrificing balance and accuracy. Given the high cost of collecting, analyzing, and interpreting metrics, balanced measurement of progress must at least encompass the dimensions of feasibility, fit for purpose, and effectiveness, and it should include both outcome and predictive KPIs.

Maintaining continuous change in any organization requires maintaining an ongoing cultural acceptance for change. Change creates fear and introduces risk that can only be overcome by a concerted effort that is supported by senior management. In the authors' experience, continuously nurturing a culture of change is especially important within the IT function because of the high rate of staff turnover in IT role assignments. The imperative to sustainably manage change across a wide range of capabilities is unavoidable for the IT function of a firm. IT executives must proactively manage the cultural acceptance for change if they wish to overcome the learned helplessness that is prevalent in most IT functions and otherwise prevents the IT function from being innovative in responding to the needs of the firm.

Given that most firms cannot stand still, if the IT function does not embrace sustainable change, new technology will be introduced anyway. The difference is that, without proactive management of capabilities, the new additions will quickly become unmanageable. 


\section{Enabling Sustainable Improvement in IT Entrepreneurship}

Paul E. Renaud, Sheppard D. Narkier, and Sonia D. Bot

\section{About the Authors}

Paul Renaud is Chief Executive of The Lanigan Group, which specializes in customer-driven product strategy and business-aligned IT service delivery. He is an advisor to CEOs, CTOs, and CIOs in the technology community, and he is a member of industry advisory boards, including Queen's University's Innovation Council for the School of Computing and Ubiquity's Chairman's Advisory Board prior to its acquisition by Avaya. His previous roles include VP Business Intelligence Development at Cognos, Director of Computing \& Networking and the Advanced Computing Research Lab at Bell Northern Research, Director of Nortel's Public Network Switching Capacity program, and Chief Architect at SHL Systemhouse. Mr. Renaud is a co-author of several patents and authored Introduction to Client/Server Systems, which was published in four languages and widely used as a university textbook. He has a BSc degree in Computer Science and Mathematics from Queen's University.

Sheppard Narkier is a business-driven, senior executive in information technology who generates business value where investment in enabling technology is an integral part of a company's business strategy. Sheppard's experience spans roles as a senior executive, enterprise architect, systems engineer, and developer. He has been recognized for building strong, diverse, and motivated teams that have delivered measurable business value in diverse IT environments. He has implemented mission-critical systems, reusable assets, and technology roadmaps in premier financial services institutions such as the American Stock Exchange, S\&P, and UBS-IB. Sheppard was co-founder and Chief Scientist of Adaptivity, which was recently acquired by EMC. Sheppard is responsible for designing the rules engines that enable better system-design decisions. Sheppard has a BA in both Mathematics and Anthropology from Oswego State, NY. He is the co-author on several patents, has written thought-leadership blogs for Network World and EMC InFocus, and has ghostwritten the book Next Generation Datacenters in Financial Services: Driving Extreme Efficiency and Effective Cost Savings.
Sonia Bot is an entrepreneurial-minded leader/executive and strategic thinker with extensive experience in technology innovation and global business management. She specializes in new venture creation, operational excellence, business transformation and strategy, leading organizational change, and evolving entrepreneurial ecosystems. She is an accomplished industry presenter, author of numerous peer-reviewed articles, member of the Lead to Win Council, and industry executive member of the Technology Entrepreneurship \& Commercialization Council at Carleton University. Ms. Bot currently partners with executives and entrepreneurs of smallto-medium enterprises and large entrepreneurial companies in the information and computing technology sector (namely healthcare, mobility, telecommunications, software, Internet, IT) to assist in building, growing, and transforming global ventures and to solve wicked business problems. Her prior work experience includes BlackBerry / Research In Motion, Nortel, Bell-Northern Research, IBM, and TransCanada Pipelines. She holds degrees in Computer Science with Systems Design / Electrical Engineering (BMath) from the University of Waterloo and Biomedical Engineering (MASc) from the University of Toronto, and she is a certified Lean Six Sigma Master Black Belt.

Citation: Renaud, P.E., S.D. Narkier, and S.D. Bot.

2013. Enabling Sustainable Improvement in IT

Entrepreneurship. Technology Innovation

Management Review. June 2013: 28-38.

Keywords: IT entrepreneurship, process ambidexterity, IT capability, process maturity, capability maturity assessment, sustainable metrics, IT function 